Document downloaded from:

http://hdl.handle.net/10251/99056

This paper must be cited as:

Riera-Guasp, M.; Fernández Cabanas, M.; J. Antonino-Daviu; Manuel Pineda-Sanchez; Rojas García, CH. (2010). Influence of non-Consecutive Bar Breakages in Motor Current Signature Analysis for the Diagnosis of Rotor Faults in Induction Motors. IEEE Transactions on Energy Conversion. 25(1):80-89. doi:10.1109/TEC.2009.2032622

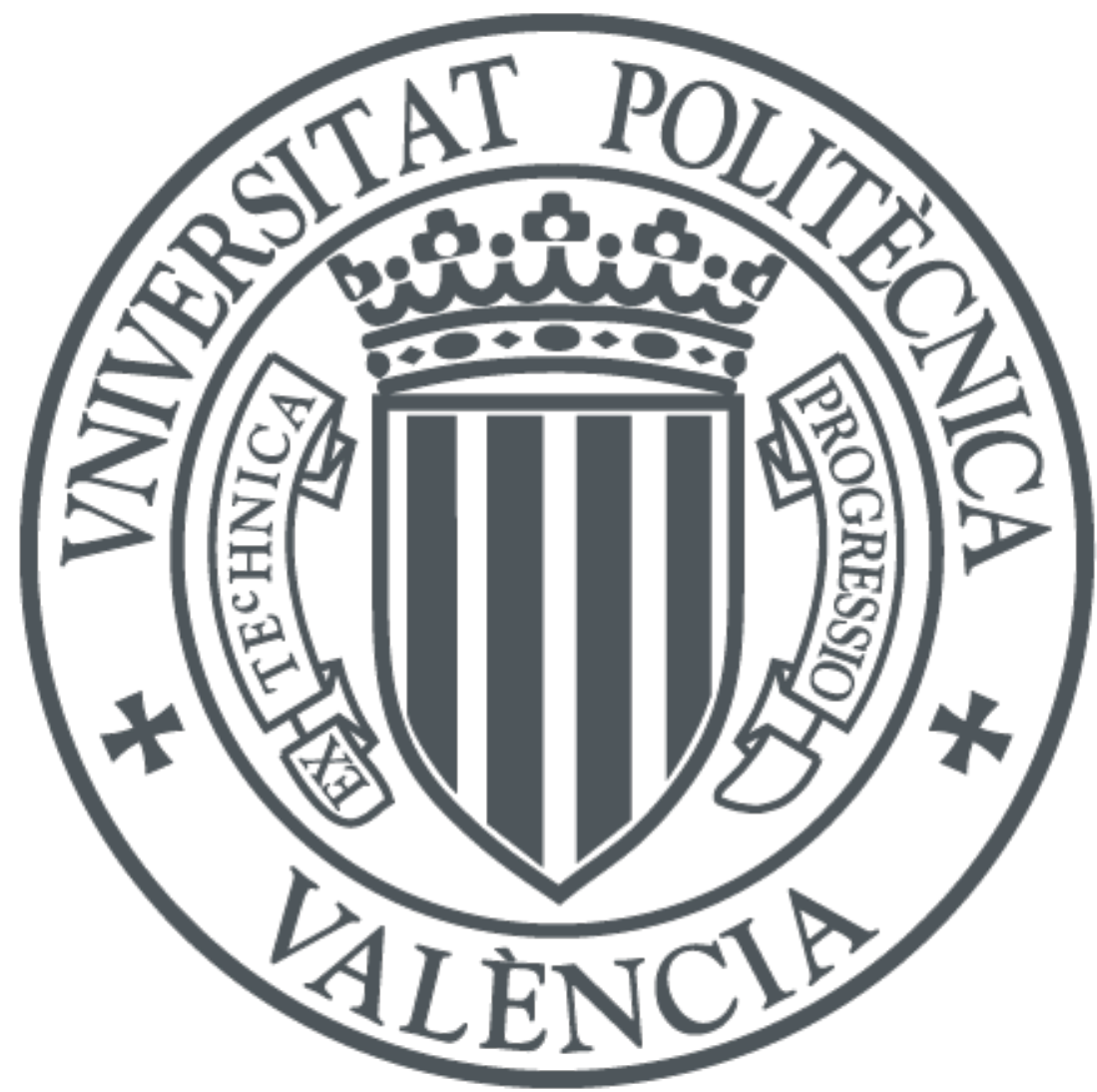

The final publication is available at

http://doi.org/10.1109/TEC.2009.2032622

Copyright Institute of Electrical and Electronics Engineers

Additional Information 


\title{
Influence of non-Consecutive Bar Breakages in Motor Current Signature Analysis for the Diagnosis of Rotor Faults in Induction Motors
}

\author{
M.Riera-Guasp, Member, IEEE, M.F.Cabanas, Member, IEEE, J.Antonino-Daviu Member, IEEE, \\ M.Pineda-Sánchez, Member, IEEE, C. H. Rojas, Member, IEEE
}

\begin{abstract}
Studies of rotor asymmetries in squirrel-cage induction motors have traditionally focused on analyses of the effects of the breakage of adjacent bars on the magnetic field and current spectrum. However, major motor manufacturers have reported cases where damaged bars are randomly distributed around the rotor perimeter of large $\mathrm{HV}$ machines. In some of these cases, the motors were being monitored under maintenance programs based on Motor Current Signature Analysis (MCSA) and the degree of degradation found in the rotor was much greater than that predicted by analysis of their current spectra. For this reason, a complete study was carried out, comprising of a theoretical analysis, as well as simulation and tests to investigate the influence that the number and location of faulty bars has on the traditional MCSA diagnosis procedure.

From the theoretical analysis, based on the application of the fault-current approach and space-vector theory, a very simple method is deduced which enables the left sideband amplitude to be calculated for any double bar breakage, per unit of the sideband amplitude corresponding to a single breakage. The proposed methodology is generalized for the estimation of the sideband amplitude in the case of multiple bar breakages and validated by simulation using a finite-element (FE) based model, as well as by laboratory tests.
\end{abstract}

Index Terms-Fault diagnosis, induction motors, broken-bar rotor faults, non-adjacent broken bars, space vector theory, motor current signature analysis (MCSA).

\section{NOMENCLATURE}

$B_{F}(\alpha, t)$ Flux density wave generated by the fault field in the air-gap

$f \quad$ Frequency of the supply voltage

$f_{s} \quad$ Sampling frequency

$f_{L s} \quad$ Frequency of the left sideband

M.Riera-Guasp, J.Antonino-Daviu and M.Pineda-Sánchez, are with the Department of Electrical Engineering, Universidad Politécnica de Valencia, Spain, e-mail: mriera@die.upv.es, joanda@die.upv.es,mpineda@die.upv.es.

M.F.Cabanas and C. H. Rojas are with the Department of Electrical Engineering, Universidad de Oviedo, Spain, e-mail: manes@uniovi.es, chrojas@uniovi.es. $i_{b i(t)} \quad$ Current in bar $b_{\mathrm{i}}$ in the healthy machine

$i_{F, b i} \quad$ Fault current in bar $b_{i}$

$\vec{i}_{F p, b i}$ Space vector of the pth order component of the fault field current density wave caused by the breakage of bar $b_{i}$

$\vec{i}_{F p-, b i}$ Space vector of the pth order inverse component of the fault field current density wave caused by the breakage of bar $b_{i}$

$\vec{i}_{F p-}$ Space vector of the resultant pth order inverse component of the fault field current density wave caused by a double or by a multiple bar breakage

$I_{f} \quad$ rms value of the fundamental component of the current current

$I_{L s, \text { double }}$ rms value of the left sideband caused by a double bar breakage

$I_{L s, \text { simple }}$ rms value of the left sideband caused by a single bar breakage

$I_{\text {Ls,multiple }}$ rms value of the left sideband caused by a multiple bar breakage

$I_{r} \quad$ rms value of the current in the rotor bars (healthy machine)

$\boldsymbol{K}_{\boldsymbol{F} \boldsymbol{p}}$ Complex factor for calculating the amplitude of the pth component of the current density wave caused by the fault field

$p \quad$ Number of pole pairs

$R \quad$ Number of rotor bars

$R_{r} \quad$ Number of broken rotor bars

$s \quad$ Slip

$\alpha_{b i} \quad$ Angular coordinate of bar $b_{i}$ in the rotor reference frame; angular distance between the second broken bar $\mathrm{b}_{\mathrm{i}}$ and the first broken bar $\mathrm{b}_{0}$

$\alpha_{s} \quad$ Rotor slot pitch

$\omega \quad$ Pulsation of supply voltage

$\omega_{r} \quad$ Pulsation of rotor bars current (healthy machine) 


\section{INTRODUCTION}

$\mathrm{R}$ eviews of maintenance reports drawn up by large companies, on-site inspections, and personal interviews with experts all confirm that failure of non-adjacent bars is fairly common in large cage asynchronous motors. Fig. 1 presents a photograph of one of the inspected motors in which the breakage of multiple rotor bars can be clearly seen.

In a relevant number of cases, the faulty motor was part of a predictive maintenance program which included periodical monitoring of the rotor using conventional MCSA tests. However, the application of current spectral analysis did not lead to an accurate diagnosis, since the degree of rotor asymmetry was incorrectly evaluated. These diagnostic errors indicate that the appearance of a motor's current spectrum is different when the faulty bars are randomly distributed around the rotor than when the bars are adjacent.

The traditional theory regarding the evolution of rotor asymmetries (bar breaking or cracking) in squirrel-cage asynchronous motors, postulates that the current layer of the rotor undergoes two increases in the bars adjacent to a breakage. Depending on the distance to the breakage [1-4] these increases are smoothly damped. However, previous studies carried out by the authors evidenced a different behaviour in the rotor current layer after a failure [5]. The rotor current layer before and after a bar breakage was analysed by applying a finite element model of an asynchronous motor. The results revealed an asymmetrical distribution of the current on both sides of the failure as well as some secondary increases that were at a distance from the breakage equal to the machine pole pitch [5]. These transient secondary increases appeared cyclically in the rotor current layer - and were considered to be a possible cause for the progressive degradation of bars that were randomly distributed around the rotor perimeter. Fig. 2 shows how the distortion in the rotor current layer after the failure is not confined to the region of the broken bar, and evolves during the electrical cycle. Secondary increases appear in the current of the bars, and are located at a distance approximately equal to the machine pole pitch [5]. Although the magnitude of these secondary effects is lower, and their presence is variable during an electrical cycle, they may cause the initiation of new bar failures at other points in the cage.

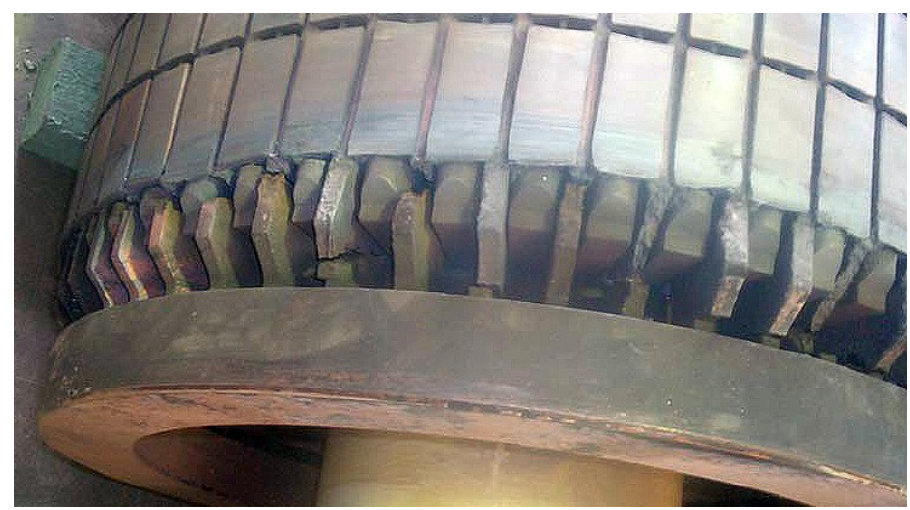

Fig 1. Rotor of a $5 \mathrm{MW}, 6 \mathrm{kV}$ cage motor with multiple broken bars.

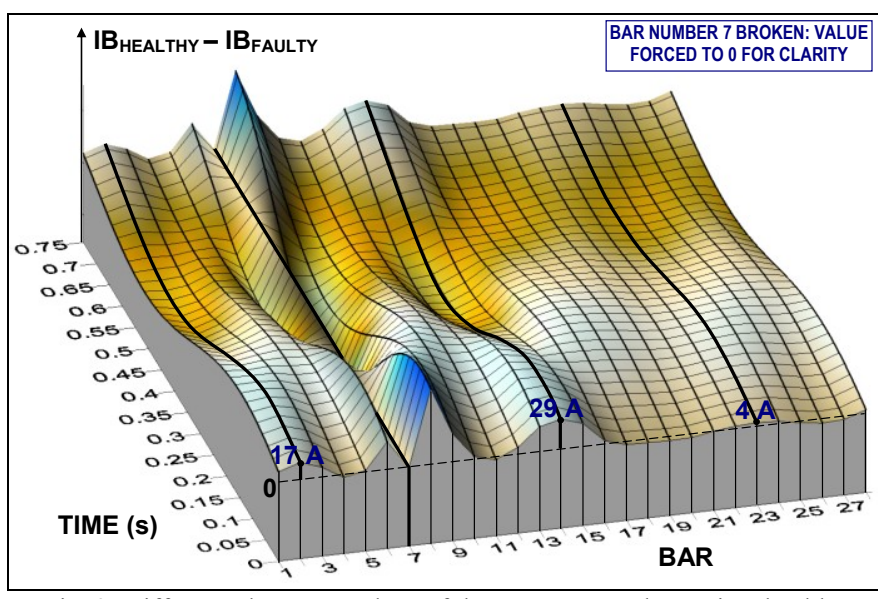

Fig. 2. Difference between values of the current rotor layers in a healthy motor, and a motor with a broken bar- during a complete electric cycle of the rotor currents. Results obtained in the simulation of an $11 \mathrm{~kW}, 4$ pole asynchronous motor.

Since the cage rotors of asynchronous machines are cylindrical, their symmetry and the presence of unavoidable manufacturing imperfections mean that there is the same likelihood of bar breakage occurring randomly anywhere in the cage. Consequently, it is not surprising that progressive damage in the rotor could start simultaneously at different points of the cage, and then evolve from each point at a different speed - depending on the thermal, magnetic and dielectric asymmetries of the machine. As a result, a bar breakage may be dispersed around the rotor perimeter.

Although comments regarding the influence of multiple bar breakages on fault diagnosis can be found since the beginning of MCSA development [6], there are few references in the literature about the diagnosis of faults involving two or more non-contiguous bars. A proposed expression for estimating the amplitude of the left sideband from the number of broken bars is found in [6], but this is restricted to the case of contiguous broken bars. These authors report that, in the case of bars fractured at intervals of $\pi / 2$ electrical radians, no significant variation in the current occurs, concluding that diagnostics based on speed or current analysis 'are liable to under-estimate the number of bars broken and may, under certain rare circumstances, fail to detect a defect'. [7] states that 'if there are broken bars in various parts of the rotor, the current analysis is not capable to provide information of the configuration of non-contiguous broken bars', remarking that 'the frequency component $f \cdot(1-2 s)$ does not exist if broken bars are electrically $\pi / 2$ radians away from each other'. Models able to analyze the behaviour of the induction motor with arbitrary bar breakages have been proposed since the 1980s, [8-9]. More recently, a model including all the cage elements (bars and end ring segments) and enabling the stator and rotor currents to be calculated for any rotor asymmetry condition was presented in [10]. This model was applied to a 22 bar, 4-pole machine, and all the possible double bar breakages were analyzed. This analysis showed that the amplitude of the left sideband harmonic varies strongly with the relative position of the broken bars. These authors state that 'for certain relative positions, the sideband amplitude is much lower than in the case of a single bar breakage and so, it 
can lead to an erroneous diagnostic of healthy machine, if conventional MCSA is used'. They conclude that a single measurement of the amplitude of the sideband 'is insufficient for the exact diagnosis of the rotor cage faults' and state that 'this component should be monitored permanently for a healthy cage to detect the beginning of a destruction process in the cage'. Some very recent works dealing with the problems of diagnosing non-adjacent broken bars can be found in [11][13]. [11] carried out an empirical analysis, based on a circuitmodel and tests, of the experimental detection of rotor faults via MCSA, and including several cases with non-adjacent broken bars. [12] simulates an induction motor using a time stepping finite element method and perform the MCSA of the simulated current for some specific cases in which four broken bars are distributed in different modes over the poles of the motor.[13] develops a thorough analysis of the induction motor with non-contiguous broken bars, including a qualitative physical interpretation, simulation and tests. The study is focused on three specific fault scenarios: adjacent broken bars; non-adjacent broken bars separated by a half-pole pitch; and non-adjacent broken bars separated by one pole pitch.

References [14]-[16] also discuss the multiple bar breakage phenomenon, but assumme that the broken bars are contiguous. [14] introduces an analytical approach that enables the analysis of the modification introduced by $n$ consecutive broken bars in the magnetomotive force, and then calculates the spectrum of the stator current, while showing the effect of the number of consecutive broken bars in the amplitude of the different fault-related harmonics. [15] and[16] compare different approaches for estimating the number of broken bars (fault severity), while also assuming that faulty bars are contiguous.

Unlike previous studies, this paper presents a physical analysis of the air-gap magnetic anomaly for the case of any double bar breakage. This analysis enables an understanding of the influence of the position of the broken bars on the diagnostic signals. Moreover, a very simple expression is deduced from this analysis, that enables the amplitude of the left sideband to be calculated for any double breakage per unit of the sideband amplitude corresponding to a single breakage. The method is also generalized for the estimation of the sideband amplitude for multiple bar breakages in which broken bars are placed in any position.

The proposed methodology is validated by simulation using a finite-element based model; and also by a significant number of laboratory tests carried out on a set of commercial cage motors.

\section{PHYSICAL ANALYSIS OF DOUBLE BREAKAGES WITH AN ARBITRARY RELATIVE POSITION OF THE BARS}

This section explains a simple method for evaluating the amplitude of the left sideband harmonic when a second bar breakage occurs as a function of the relative position of the broken bars. The method is based on the physical analysis of the air-gap anomaly using the fault-current approach [1] and the space vector theory $[17,18]$.

The effects of a broken bar can be analysed using the concept of fault-current presented by Deleroi [1]. This means that a machine with a broken rotor bar may be analysed by considering the superposition of two configurations: the machine in a healthy state; and the machine with a current source placed in the bar that breaks (fault current). The fault current is always equal to the current flowing through the same bar in the healthy machine but in the opposite direction, - so that the total current through the broken bar is null. At steady state, the fault current varies sinusoidally with time at the slip frequency. Let us assume that bar $b_{0}$ breaks; selecting a suitable time origin, the expression for the fault current is:

$$
i_{F, b 0}=-i_{b 0}=\sqrt{2} \cdot I_{r} \cdot \cos \left(\omega_{r} t\right)
$$

where $i_{F, b 0}, i_{b 0}$ denote, respectively, the fault current and the current in the healthy machine through the bar $b_{0} ; \omega_{r}, I_{r}$ are, respectively, the pulsation and rms values of the rotor currents for a given slip $s$, in the healthy machine:

$$
\omega_{r}=s \omega=2 \pi s f
$$

$\omega$ and $f$ respectively being the pulsation and frequency of the supply voltage.

The fault current flows through the short-circuit rings and the remaining bars, creating a magnetic field in the air-gap (fault field). This field, superimposed on the normal field of the healthy machine, causes alterations in its behaviour. More specifically, it induces the current harmonics in the stator windings that are used for the bar breakage diagnosis.

The shape of the spatial wave of air-gap flux density $B_{F}(\alpha, t)$ caused by the fault field is a stepped bipolar wave, the amplitude and spectral composition of which vary with time. As stated in [1, 19], the fault field may be decomposed as the sum of spatial harmonics with $1,2,3 . . p . . n$ pole pairs. Although these harmonics have fixed positions with respect to the rotor, their amplitudes oscillate proportionally to the fault current. From this point onwards, the analysis will focus on the component of the fault field with $p$ pole pairs, since this component generates the left sideband harmonic. Using the formulation of space vector theory [17-18], the space vector of the $\mathrm{p} t h$ component of the current density wave generated by the fault field ( $\mathrm{p} t h$ current space vector) is deduced in [20], obtaining:

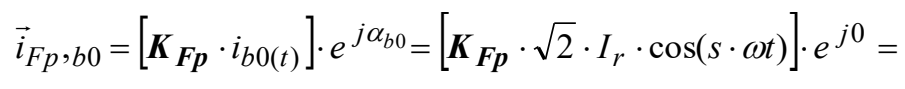

$\left[\boldsymbol{K}_{\boldsymbol{F p}} \cdot \sqrt{2} \cdot I_{r} \cdot\left(\frac{e^{j s \omega t}+e^{-j s \omega t}}{2}\right)\right] \cdot e^{j 0}$

The expression within brackets is the magnitude of the vector, which varies in time proportionally to the fault current. Its argument is constant, and it coincides with the coordinate 
of $b_{0}$ (in electrical radians), which was selected as the origin of angular coordinates $\left(\alpha_{b 0}=0\right) . \boldsymbol{K}_{\boldsymbol{F}}$ is a complex factor deduced in [20] which depends on the characteristics and slip of the machine.

As clearly shown by (3), the pth order current density wave may be decomposed as the sum of two rotating components, direct and inverse, with constant amplitude and slip (Leblanc's theorem). The left sideband harmonic is caused by the inverse component, the space vector of which is:

$$
\vec{\imath}_{F p-, b 0}=\boldsymbol{K}_{\boldsymbol{F p}} \cdot \frac{\sqrt{2}}{2} \cdot I_{r} \cdot e^{-i s \omega t} \cdot e^{j 0}
$$

When a second bar $b_{i}$ breaks, a new fault field appears. If $\alpha_{b i}$ is the geometric angle between both broken bars, the pth current space vector generated by the second broken bar is given by:

$$
\begin{gathered}
\vec{i}_{F p, b i}=\boldsymbol{K}_{\boldsymbol{F p}} \cdot i_{b i(t)} \cdot e^{j p \alpha_{b i}}= \\
=\left[\boldsymbol{K}_{\boldsymbol{F p}} \cdot \sqrt{2} \cdot I_{r} \cdot \cos \left(s \cdot \omega t-p \alpha_{b i}\right)\right] \cdot e^{j p \alpha_{b i}}= \\
=\left[\boldsymbol{K}_{\boldsymbol{F p}} \cdot \sqrt{2} \cdot I_{r} \cdot\left(\frac{e^{j\left(s \omega t-p \alpha_{b i}\right)}+e^{-j\left(s \omega t-p \alpha_{b i}\right)}}{2}\right)\right] \cdot e^{j p \alpha_{b i}}
\end{gathered}
$$

Equation (5) is directly deduced from (3), taking into account the following comments:

As in the previously mentioned case of bar $b_{0}$, the amplitude of this vector (within brackets) is proportional to the fault current running through the bar $b_{i}$, and the fault current is equal to the current through this bar in the healthy machine but with the opposite sign. Since the currents running through $b_{0}$ and $b_{i}$ are induced by the same rotating field (the main sinusoidal air-gap rotating field of the machine), the current in $b_{i}$ has the same amplitude than as $b_{0}$ but is delayed by an angle $p \alpha_{b i}$. This is equal to the angular distance between both bars, expressed in electrical radians. As deduced in [17], the argument of the space vector of current density generated by a simple conductor (bar $b_{i}$ ) coincides with the angular coordinate of the conductor in electrical radians.

Fig. 3 (a) shows a cross-section of the rotor after the second breakage, in which the pth order current density wave components of the fault fields created by both broken bars at a given time are indicated. Fig.3 (b) shows the corresponding space vector diagram. The space vectors corresponding to both broken bars keep their relative position constant; their amplitudes oscillate with time with the same pulsation $s \omega$, but with different time phases (reaching their maxima at different times).

In the case of double bar breakages, the left sideband harmonic is produced by the resultant $\mathrm{p}$ th order inverse component of the fault field. The current space vector of the pth inverse component of the fault field produced by the second broken bar is given by (6):

$$
\begin{gathered}
\vec{i}_{F p-, b i}=\boldsymbol{K}_{\boldsymbol{F p}} \cdot \frac{\sqrt{2}}{2} \cdot I_{r} \cdot e^{-j\left(s \omega t-p \alpha_{b i}\right)} \cdot e^{j p \alpha_{b i}=} \\
=\vec{i}_{F p-, b 0} \cdot e^{j 2 p \alpha_{b i}}
\end{gathered}
$$

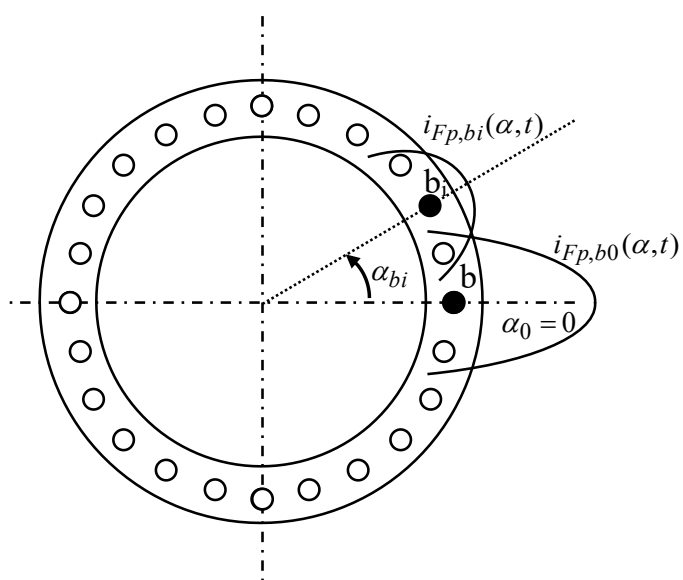

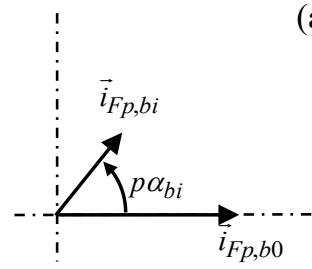

(b) (a)

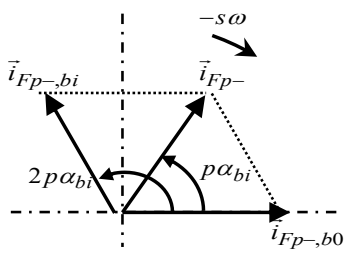

(c)
Fig. 3. The pth current density component of the fault field in a double bar breakage: (a) rotor cross-section, (b) space vector diagram. (c) vector representation of (7).

The space vector of the resultant $p t h$ order inverse current density wave component of the fault field is the sum of (4) and (6):

$$
\vec{i}_{F p-}=\vec{i}_{F p-, b 0}+\vec{i}_{F p-, b i}=\vec{i}_{F p-, b 0} \cdot\left(1+e^{j 2 p \alpha_{b i}}\right)
$$

Fig. 3 (c) shows the vector representation of (7). The space vectors corresponding to both broken bars have constant and identical amplitudes. Their relative position is also constant, since all the vectors of the diagram rotate with respect to the rotor at the constant speed $-s \omega$. Their arguments differ in an angle equal to twice the electrical angle between both bars. This double displacement is caused by the combination of the spatial angular separation of the bars ( $p \alpha_{b i}$ electrical radians) and the time delay between the fault currents that circulate through them $\left(\omega t=p \alpha_{b i}\right)$.

The ratio between the amplitudes of the left sideband harmonics in the cases of double and single bar breakages coincides with the ratio between the amplitudes of the pth inverse flux density components of the fault field. From an approximate analysis, if saturation is neglected, this ratio is in agreement with that between the current density components, and finally resulting in:

$$
\begin{gathered}
i_{\text {Ls, double pu }}=\left|\frac{I_{\text {Ls, double }}}{I_{\text {Ls, simple }}}\right| \approx\left|\frac{\vec{i}_{F p-}}{\vec{i}_{F p-, b 0}}\right|= \\
=\mid 1+e^{j 2 p \alpha_{b i}|=| 2 \cdot \cos \left(p \alpha_{b i}\right) \mid}
\end{gathered}
$$


This expression enables the variation of the left sideband to be estimated when a second bar breaks, as a function of the relative position of the two broken bars. Fig. 4 shows the evolution of $i_{L s, d o u b l e, p u}$ as a function of $\alpha_{b i}$ deduced from (8). The sideband amplitude is given per unit of its amplitude for a single breakage; and the graphic is particularized for a fourpole machine.

Observation of Fig. 4 leads to the following conclusions:

- If the positions of the broken bars differ by exactly an entire number of pole pitches $\left(\alpha_{b i}=k \cdot \pi / p \quad k=1,2,3 \ldots.\right)$, the amplitude of the left sideband doubles that corresponding to a single breakage (relative amplitude $i_{L s, \text { double }}=2$ ).

- If $\alpha_{b i}$ is small (as in the case of consecutive bar breakages) or the position of the broken bars differs slightly from an entire number of pole pitches $\left(\alpha_{b i} \approx k \cdot \pi / p\right.$ $k=0,1,2,3 \ldots)$, then the relative amplitude between left sidebands is slightly lower than 2 .

- If $\alpha_{b i}$ approximates $\pi / 3 p$ or $2 \pi / 3 p$, or the distance between both broken bars is approximately equal to these angles plus an entire number of pole pitches $\left(\alpha_{b i} \approx \pi / 3 p+k \cdot \pi / p\right.$ or $\left.\alpha_{b i} \approx 2 \pi / 3 p+k \cdot \pi / p ; k=0,1,2,3 \ldots\right)$, then the amplitude of the left sideband is practically the same as that for the single breakage. Hence, the second bar breakage cannot be detected by analyzing the left sideband.

- If $\alpha_{b i}$ approximates $\pi / 2 p$ (half a pole pitch) or the distance between both broken bars is approximately equal to this angle plus an entire number of pole pitches $\left(\alpha_{b i} \approx \pi / 2 p+\right.$ $k \cdot \pi / p ; k=0,1,2,3 \ldots)$, then the second breakage reduces the amplitude of the left sideband to below that corresponding to a single breakage - and so a machine with two broken bars could be erroneously diagnosed as a healthy machine. This result, already reported by $[6,8,10,11$, 13], is due to the fact that the inverse components of the fault fields provoked by both bars are practically opposed.

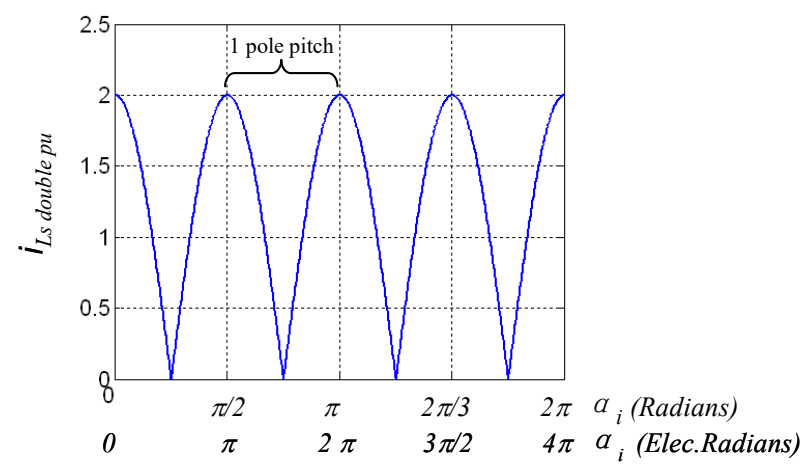

Fig. 4. Evolution of the amplitude of the left sideband harmonic in a double bar breakage as a function of the relative position of the bars (four-pole machine).
The previous remarks are applicable to machines with any number of poles and rotor bars. They are consistent with the results reported in [10] and [13]; in [10] the variation of the left sideband amplitude is obtained as a function of the position of the second broken bar, for a 4-pole machine with 22 rotor bars, using a numerical model. In [13] the amplitude of the left sideband is obtained by testing a 6-pole machine with 45 rotor bars and with two broken bars spaced at 4 slots.

For a specific machine, with a given number $R$ of rotor bars and $p$ pole pairs, the angular distance between the broken bars may be calculated as:

$$
\alpha_{b i}=i \cdot \alpha_{s}=i \cdot \frac{2 \pi}{R}
$$

where $\alpha_{s}$ is the slot pitch in radians and $i$ is the number of slot pitches between both broken bars.

In the following sections, (8) is validated using numerical simulations and laboratory tests.

\section{NUMERICAL SIMULATION OF AN INDUCTION MOTOR WITH DIFFERENT DOUBLE BAR BREAKAGES.}

In this section, a bi-dimensional finite-element based model of the cross-section of an $11 \mathrm{~kW}$ industrial motor is used to simulate the behaviour of the machine with double bar breakages in different relative positions. This model, developed in previous studies by the authors [5], is able to reproduce the transient operation of the machine by means of a rotating finite element mesh, and taking into account the saturation of the magnetic circuit, thus enabling the analysis of broken rotor bars and inter-turn short-circuits, [21]. Table I presents the main characteristics of the induction motor used for the simulations. A set of simulations of double bar breakages were developed that cover all the relative positions between both broken bars in a pole pitch, as well as two cases in which the distance between broken bars was greater than a pole pitch. To serve as a reference, the case of a single breakage is also analyzed.

TABLE I. MOTOR CHARACTERISTICS

\begin{tabular}{|cc|}
\hline Number of poles & 4 \\
Rated power & $11 \mathrm{~kW}$ \\
Rated voltage & $400 \mathrm{~V}$ \\
Rated current & $22.5 \mathrm{~A}$ \\
Full-load speed & $1460 \mathrm{rpm}$ \\
Number of rotor bars & 28 \\
Number of stator slots & 36 \\
Type of rotor & Die cast-Al \\
Type of magnetic sheet & AISI- code M-36 \\
\hline
\end{tabular}

The first step for developing each simulation consists of 'breaking' the selected bars in the model by increasing their resistance to $R_{\text {broken }}=10 \mathrm{M} \Omega$. The machine is then started up direct on-line, driving a load with a torque characteristic proportional to the speed. This characteristic was arranged so that when steady state is reached, the machine works 
practically at full load $(s \approx 0.027)$. Each simulation lasted six seconds of motor operation; the time increment used for the numeric integration was $\Delta t=0.0005 \mathrm{~s}$. Fig. 5 (a) shows the evolution of the phase A stator current and speed during the first second after the connection, for the case of a single breakage.

Fig. 5 (b) shows the spectrum of the simulated current. This spectrum was calculated by applying FFT with a Hanning window to the stationary portion of the simulated current (last five seconds of the register). The left sideband can be clearly observed with a frequency $f_{L s} \approx 47.5 \mathrm{~Hz}$.

Table II summarizes the results of the simulations carried out. Column 4 shows that the amplitude of the fundamental component is practically unaffected by the position of the broken bars. Column 6 gives the amplitude of the left sideband obtained for the different faults specified in Column 1, per unit of the corresponding amplitude for a single breakage. The last column also gives this value, though calculated theoretically using (8). It has to be remarked that since a perfect symmetry is assumed in the healthy machine, the double breakage $\left(\mathrm{b}_{0}-\mathrm{b}_{\mathrm{i}}\right)$ is equivalent to the breakage $\left(\mathrm{b}_{0}-\mathrm{b}_{28-\mathrm{i}}\right)$.

Fig. 6 compares Column 6 of Table II with the values of $I_{L s}$ calculated from (8). The figure clearly shows that the variation of the left sideband amplitude versus the relative position of both broken bars, calculated using the FE model, fits well the variation predicted by the simplified equation (8) deduced in Section II.

Fig. 5. (a) phase A stator current and speed (simulated), (b) Spectrum of the

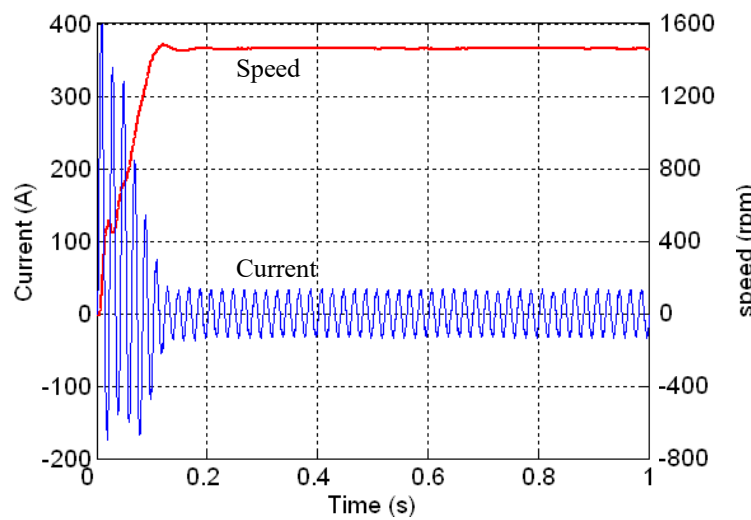

(a)

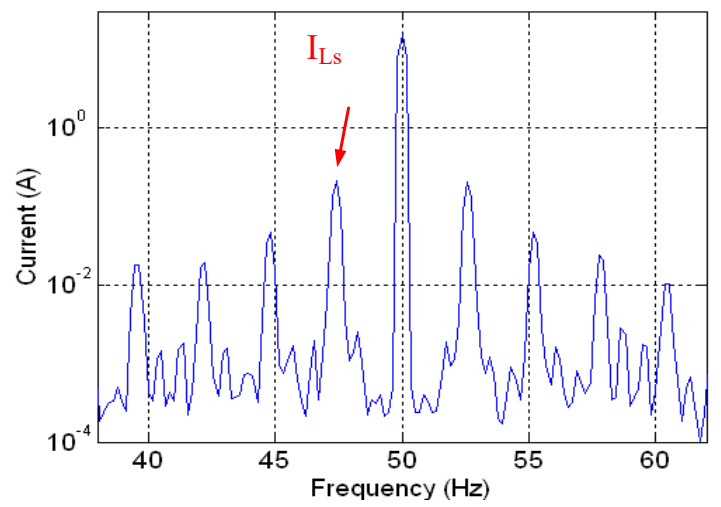

(b)

simulated phase A current.
TABLE II

RESULTS OF THE SIMULATIONS

\begin{tabular}{|c|c|c|c|c|c|c|}
\hline Broken bars & $\begin{array}{c}\text { Geometric } \\
\text { Distance } \\
\alpha_{b i} \\
\text { (degrees) }\end{array}$ & $\begin{array}{c}\text { Electrical } \\
\text { Distance } \\
\boldsymbol{p} \boldsymbol{\alpha}_{b i} \\
\text { (electrical } \\
\text { degrees) }\end{array}$ & $\begin{array}{c}\text { Fundamental } \\
\text { harmonic } \\
\text { a mplitude } \\
\boldsymbol{I}_{\boldsymbol{f}}(\boldsymbol{A})\end{array}$ & $\begin{array}{c}\text { Left } \\
\text { sideband } \\
\text { Amplitude } \\
\boldsymbol{I}_{\boldsymbol{L}}(\boldsymbol{A})\end{array}$ & $\begin{array}{c}\left|\frac{I_{L s}}{I_{L s, \text { single }}}\right| \\
\text { FEM } \\
\text { simulation }\end{array}$ & $\begin{array}{c}\left|\frac{I_{L s}}{I_{L s, \text { single }}}\right| \\
\text { Theoretical } \\
\text { Eq(8) }\end{array}$ \\
\hline Healthy & - & - & 15.26 & 0.053 & 0.25 & 0 \\
\hline $\mathbf{b}_{0}$ & - & - & 15.32 & 0.215 & 1.00 & 1.00 \\
\hline$\left(\mathrm{b}_{0}-\mathrm{b}_{1}\right) \equiv\left(\mathrm{b}_{0}-\mathrm{b}_{27}\right)$ & 12.85 & 25.71 & 15.63 & 0.409 & 1.90 & 1.80 \\
\hline$\left(\mathrm{b}_{0}-\mathrm{b}_{2}\right) \equiv\left(\mathrm{b}_{0}-\mathrm{b}_{26}\right)$ & 25.71 & 51.43 & 15.50 & 0.274 & 1.28 & 1.25 \\
\hline$\left(\mathrm{b}_{0}-\mathrm{b}_{3}\right) \equiv\left(\mathrm{b}_{0}-\mathrm{b}_{25}\right)$ & 38.57 & 77.14 & 15.38 & 0.099 & 0.46 & 0.45 \\
\hline$\left(\mathrm{b}_{0}-\mathrm{b}_{4}\right) \equiv\left(\mathrm{b}_{0}-\mathrm{b}_{24}\right)$ & 51.42 & 102.86 & 15.56 & 0.081 & 0.37 & 0.45 \\
\hline$\left(\mathrm{b}_{0}-\mathrm{b}_{5}\right) \equiv\left(\mathrm{b}_{0}-\mathrm{b}_{23}\right)$ & 64.28 & 128.57 & 15.48 & 0.332 & 1.54 & 1.25 \\
\hline$\left(\mathrm{b}_{0}-\mathrm{b}_{6}\right) \equiv\left(\mathrm{b}_{0}-\mathrm{b}_{22}\right)$ & 77.14 & 154.29 & 15.56 & 0.381 & 1.77 & 1.80 \\
\hline$\left(\mathrm{b}_{0}-\mathrm{b}_{7}\right) \equiv\left(\mathrm{b}_{0}-\mathrm{b}_{21}\right)$ & 90 & 180.00 & 15.41 & 0.390 & 1.81 & 2.00 \\
\hline$\left(\mathrm{b}_{0}-\mathrm{b}_{11}\right) \equiv\left(\mathrm{b}_{0}-\mathrm{b}_{17}\right)$ & 192.85 & 385.71 & 15.55 & 0.411 & 1.91 & 1.80 \\
\hline$\left(\mathrm{b}_{0}-\mathrm{b}_{13}\right) \equiv\left(\mathrm{b}_{0}-\mathrm{b}_{15}\right)$ & 218.57 & 437.14 & 15.72 & 0.093 & 0.43 & 0.44 \\
\hline
\end{tabular}

\section{EXPERIMENTAL STUDY OF DOUBLE BAR BREAKAGES}

A series of laboratory tests were subsequently carried out using commercial motors in order to confirm the validity of the preceding theoretical analysis for industrial machines.

Seven identical cage motors rated $1.1 \mathrm{~kW}, 400 \mathrm{~V}$ (star), with 4 poles and 28 rotor bars were used for the tests. The motors were disassembled; one of the stators was mounted on the test bed (see Fig. 7), and then a series of tests were performed by mounting the various rotors under different fault conditions.

For the initial series of tests, all the seven rotors in healthy condition were successively mounted and tested. The motor was loaded using a D.C. machine so that the rated regime ( $n=1410 \mathrm{rpm}, s=0.06$ ) was reached. Subsequently, a register of a stator phase current was obtained by means of a digital oscilloscope through a shunt $(6 \mathrm{~A}, 60 \mathrm{mV})$ connected to the secondary of a current transformer (15/5). The current signal was captured during fifty seconds, using a sampling frequency $f_{s}=5000 \mathrm{samples} / \mathrm{s}$. The aim of these tests was to confirm that there were no significant differences between the tested rotors.

After the first series of tests, a bar was broken in each of the rotors $\left(b_{0}\right.$ bar). This was achieved by drilling at the junction between the short circuit ring and the bar.

A second series of tests was then carried out following the same procedure, and using the rotors with a broken bar. These tests enabled the calculation of the amplitude of the left sideband $I_{L s, \text { single }}$ using the fast Fourier transform, for the case of a single breakage in every rotor.

Subsequently, a second bar was broken in each rotor. In order to study all the possible relative positions of both broken bars in a pole pitch, the second drilled bar was in a different position in each rotor,

Finally, a third series of tests was performed by testing the rotors with double bar breakages and calculating the corresponding left sideband amplitude $\left(I_{L s, d o u b l e}\right)$. Table III summarizes the FFT analysis of the tested currents. To reduce 


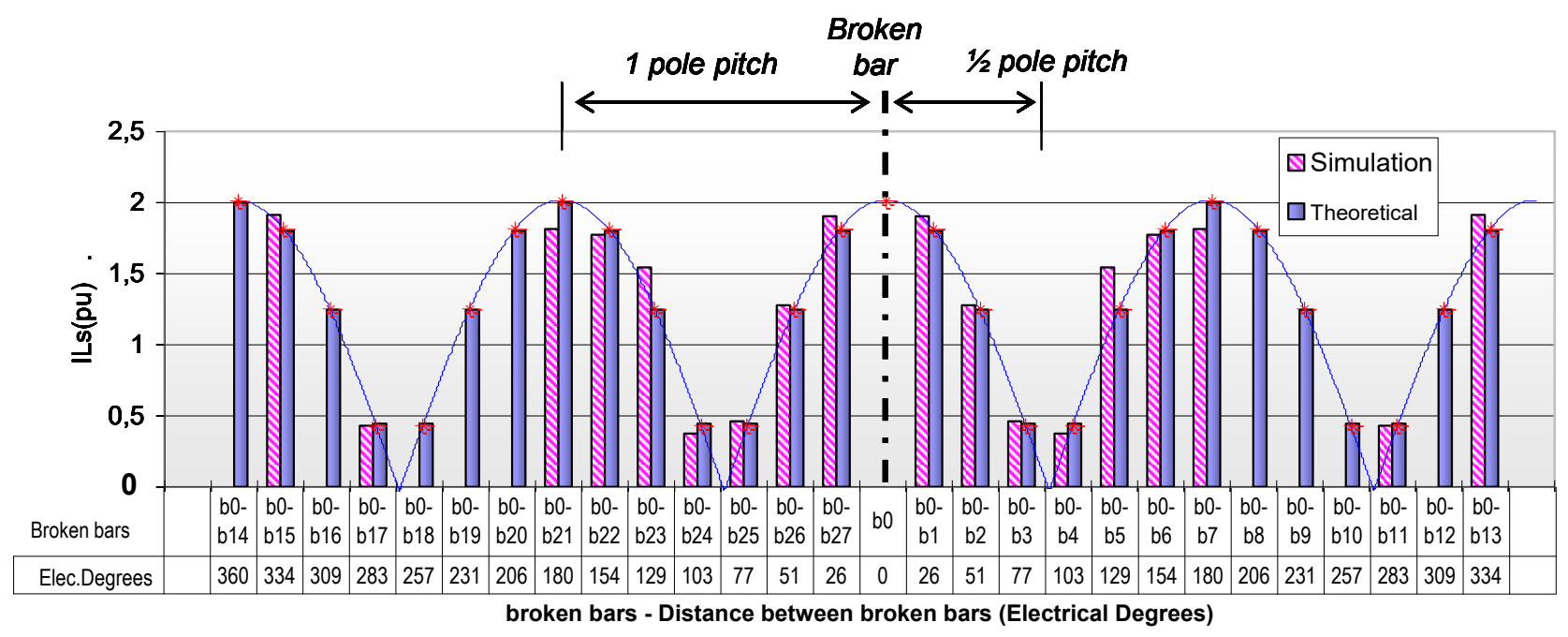

Fig. 6. Variation of the left sideband amplitude in a double bar breakage with the relative position of the broken bars (simulation and theoretical).

the influence of using seven different rotors, the amplitudes of the left sidebands were normalized by dividing them by the amplitude of the corresponding fundamental harmonic $\left(I_{f}\right)$ as measured in the same test. Column 7 gives the experimental amplitude of the normalized left sideband component for different double breakages p.u. of the sideband amplitude corresponding to the same rotor with one broken bar. Column 8 shows the theoretical values expected for these cases, calculated from (8). Fig. 8 graphically compares the tested values (Column 7 of Table III) with the theoretical values predicted by (8) and with the values obtained from the numerical simulation (Column 6 of Table II).

As expected, Table III and Fig. 8 show greater discrepancies between tested and theoretical amplitudes of the sideband

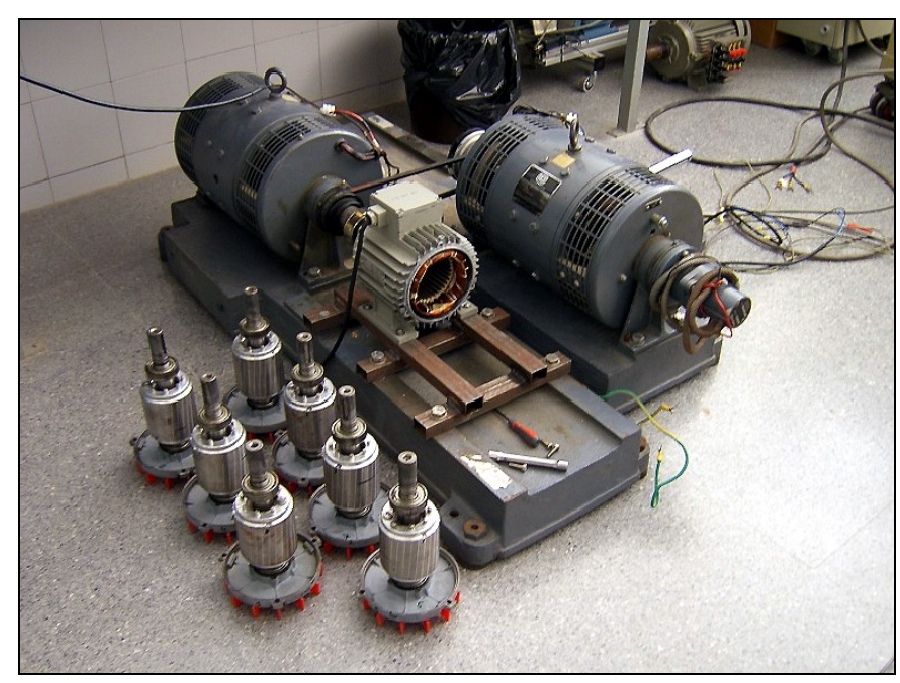

Fig. 7. Test bed and rotors used in the tests.
TABLE III.

RESULTS OF THE TESTS

\begin{tabular}{|c|c|c|c|c|c|c|c|}
\hline $\begin{array}{l}\text { Rotor } \\
\text { Number }\end{array}$ & Broken bars & $\begin{array}{c}\text { Geometric } \\
\text { Distance } \\
\alpha_{b i} \\
\text { (degrees) }\end{array}$ & $\begin{array}{c}\text { Electrical } \\
\text { Distance } \\
p^{\alpha} \boldsymbol{b i}_{\boldsymbol{i}} \\
\text { (electric. } \\
\text { degrees) }\end{array}$ & 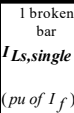 & 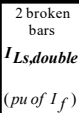 & 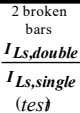 & $\mid \begin{array}{l}\left|\frac{I_{L s}}{I_{L s, s i n g l e}}\right| \\
\text { (theoreticd (8) }\end{array}$ \\
\hline 1 & $\left(b_{0}-b_{1}\right) \equiv\left(b_{0}-b_{27}\right)$ & 12.85 & 25.71 & 0,0143 & 0,0217 & 1,5198 & 1.80 \\
\hline 2 & $\left(b_{0}-b_{2}\right) \equiv\left(b_{0}-b_{26}\right)$ & 25.71 & 51.43 & 0,0213 & 0,0234 & 1,0980 & 1.25 \\
\hline 3 & $\left(b_{0}-b_{3}\right) \equiv\left(b_{0}-b_{25}\right)$ & 38.57 & 77.14 & 0,0115 & 0,0087 & 0,7527 & 0.45 \\
\hline 4 & $\left(b_{0}-b_{4}\right)_{4} \equiv\left(b_{0}-b_{24}\right)$ & 51.42 & 102.86 & 0,0081 & 0,0035 & 0,4358 & 0.45 \\
\hline 5 & $\left(b_{0}-b_{5}\right) \equiv\left(b_{0}-b_{23}\right)$ & 64.28 & 128.57 & 0,0131 & 0,0128 & 0,9827 & 1.25 \\
\hline 6 & $\left(\mathrm{~b}_{0}-\mathrm{b}_{6}\right)=\left(\mathrm{b}_{0}-\mathrm{b}_{22}\right)$ & 77.14 & 154.29 & 0,0087 & 0,0124 & 1,4245 & 1.80 \\
\hline 7 & $\left(b_{0}-b_{7}\right) \equiv\left(b_{0}-b_{2}\right)^{2}$ & 90 & 180.00 & 0,0201 & 0,0348 & 1,7372 & 2.00 \\
\hline
\end{tabular}

component than those observed for the simulations. This is due to the constructive and assembly tolerances of the different rotors; as well as the unavoidable variations in test conditions (source voltage, winding temperatures, measuring errors, etc.) during the series of tests. Nonetheless, the results of the tests clearly follow the theoretical trend. The sideband amplitude decreases as the second broken bar moves away from the first; reaching a minimum when both bars are separated by approximately half the pole pitch.

The amplitude increases from this point until reaching a maximum, when the bars are separated a pole pitch. For this case, the amplitude of the sideband practically doubles its amplitude for the single breakage. As predicted by (8), when both broken bars are separated by approximately half the pole pitch, the sideband amplitude is clearly lower than that corresponding to a single bar breakage; and the machine in this faulty condition might be wrongly diagnosed as healthy. Therefore, the simplified analysis developed in Section III proves to be useful for predicting and understanding the effect of a second bar breakage in the tested commercial motor. 


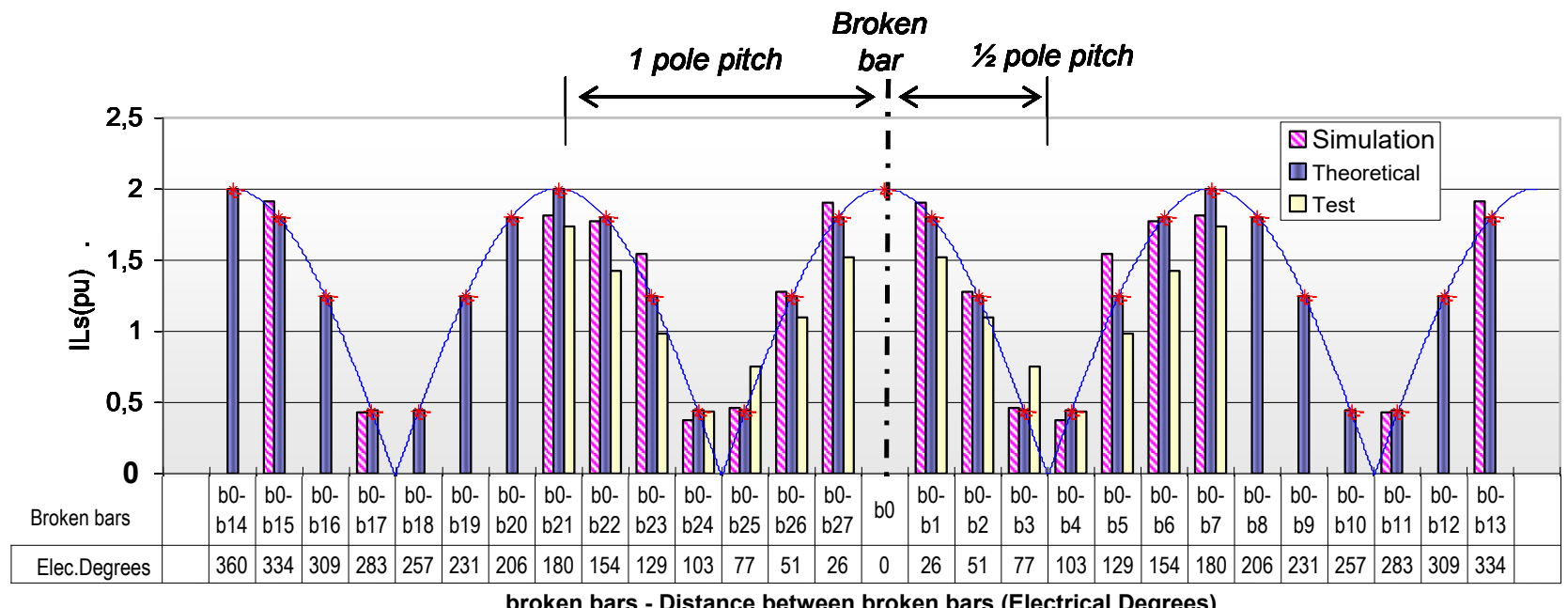

Fig. 8. Variation of the left sideband amplitude in a double bar breakage with the relative position of the broken bars (experimental, theoretical and simulated).

\section{APPROXIMATE STUDY OF MULTIPLE BAR BREAKAGES}

The simplified analysis based on the current density vectors given in Section III may be easily extended to a greater number of broken bars. The resultant vector of current density for the fault field component that creates the left sideband can be obtained by applying the superposition principle, and adding the individual components generated by each broken bar.

If bars $b_{a}, b_{b}, b_{c} \ldots$, placed at coordinates $\alpha_{b a}, \alpha_{b b}, \alpha_{b c}$ $\ldots$, are broken, the pth current space vector of the inverse component of the fault field produced by all the broken bars is given by:

$$
\begin{aligned}
& \vec{i}_{F p-}=\vec{i}_{F_{p}-, b_{a}}+\vec{i}_{F p-, b_{b}}+\vec{i}_{F p-, b_{c}}+\ldots= \\
& =\vec{i}_{F p-, b 0} \cdot\left(e^{j 2 p \alpha_{b a}}+e^{j 2 p \alpha_{b b}}+e^{j 2 p \alpha_{b c}}+\ldots\right)
\end{aligned}
$$

Thus, if saturation is neglected, the ratio between the amplitudes of the left sideband harmonics in the cases of multiple and single bar breakages is approximately given by:

$$
\begin{gathered}
i_{L s, \text { multiple } p u}=\left|\frac{I_{\text {Ls, multiple }}}{I_{\text {Ls, single }}}\right| \approx\left|\frac{\vec{i}_{F p-}}{\vec{i}_{F p-, b 0}}\right|= \\
=\left|\left(e^{j 2 p \alpha_{b a}}+e^{j 2 p \alpha_{b b}}+e^{j 2 p \alpha_{b c}}+\ldots\right)\right|
\end{gathered}
$$

To validate (11), a new series of tests were carried out, using the rotor named as 7 in Table III. Bars $b_{0}, b_{7}, b_{3}, b_{11}, b_{14}, b_{17}$, $b_{19}, \quad b_{20}, \quad b_{23}$ were successively broken and, after every breakage, the rotor was reassembled and the stator current analyzed. Fig. 9 compares the theoretical evolution of the left sideband amplitude calculated from (11), with the test results. The evolution of the sideband with the successive breakages is correctly predicted, even for a high number of broken bars. Obviously, when the number of broken bars increases, the hypothesis of the fault current proposed by Deleroi fits less well to the phenomena taking place in the machine and the analysis error increases. Fig. 10 represents the vector diagram of (11) corresponding to the first four cases of multiple breakages - and explains the evolution of the sideband amplitude when bars $b_{0}, b_{3}, b_{7}$ and $b_{11}$ are successively broken.

Finally, the experimental results reported in [13] enabled us to make a confirmation of (11) on a machine that was very different from the machine used for the tests. In [13], the authors tested a 5-hp machine ( $p=3$ pole-pairs, $R=45$ rotor bars) at rated load, under six different fault conditions:

- Healthy

- One broken bar

- Two adjacent broken bars

- Two broken bars separated by half pole-pitch

- Three broken bars each separated one half pole-pitch

- Two broken bars separated by one pole-pitch.

Equation (11) was applied (with $p=3, \alpha_{b i}=i \cdot 2 \pi / 45$ ), to the last four cases, and the corresponding normalized values of the theoretical LSH in dB, was calculated. Fig. 11 compares these theoretical values with the corresponding experimental values measured in [13], showing a good agreement.

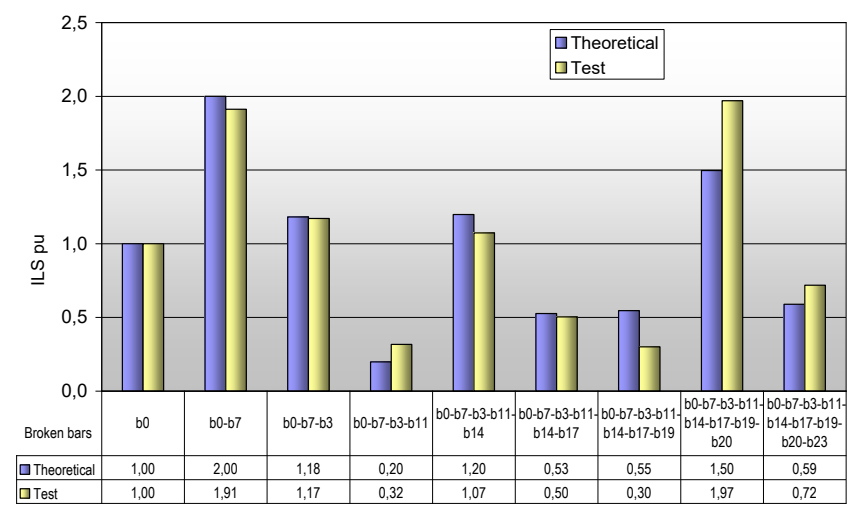

Fig. 9. Evolution of the left sideband amplitude after the successive breakages of bars $b_{0}, b_{7}, b_{3}, b_{11}, b_{14}, b_{17}, b_{19}, b_{20}, b_{23}$ (not adjacent bars). 


\section{$\overrightarrow{\vec{i}_{F p-, b 0}}$}

(a)

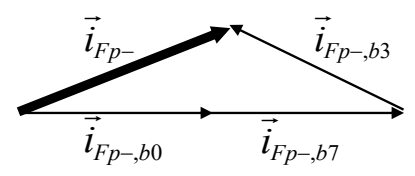

(c)

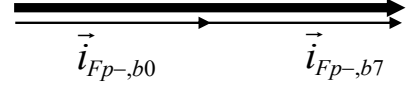

(b)

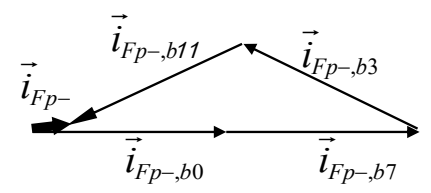

(d)
Fig. 10. Evolution of resultant current density space vector when bars $b_{0}$, $\mathrm{b}_{3,} \mathrm{~b}_{7}$ and $\mathrm{b}_{11}$ are successively broken: $(a) \mathrm{b}_{0}$ broken, $(b) \mathrm{b}_{0}, \mathrm{~b}_{7}$ broken, $(c) \mathrm{b}_{0}$, $\mathrm{b}_{7}, \mathrm{~b}_{3}$ broken, $(d) \mathrm{b}_{0}, \mathrm{~b}_{7}, \mathrm{~b}_{3}, \mathrm{~b}_{11}$ broken.

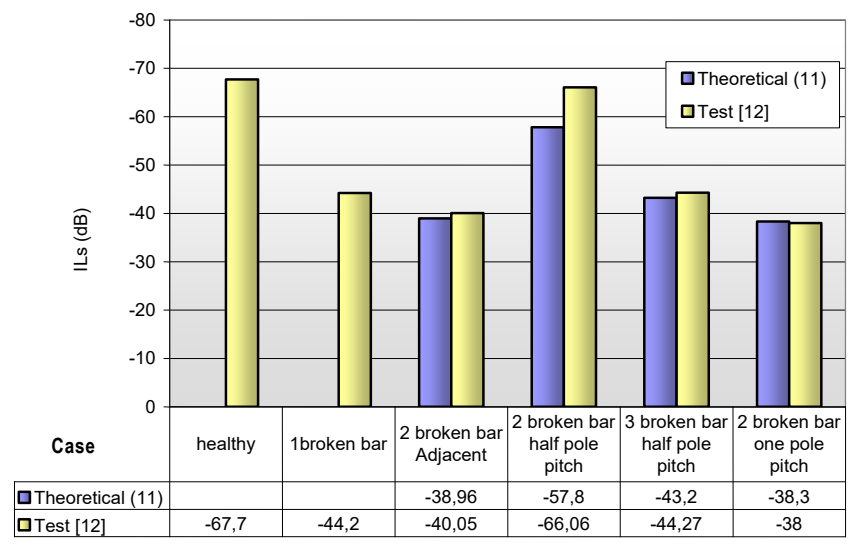

Fig. 11. Comparison of the theoretical LSH calculated through (11) with the corresponding experimental ones tested in [13] for four different cases of adjacent and nonadjacent breakages

\section{CONCLUSIONS}

An analysis of double and multiple bar breakages valid for cage motor faults involving non-consecutive broken bars has been presented. This analysis is based on the application of the fault-current approach and space vector theory. It provides a physical interpretation of the appearance of the left sideband component under any double or multiple bar breakage condition, as well as the deduction of very simple expressions for the approximate calculation of the left sideband amplitude - as a function of the relative position of the broken bars. The theoretical analysis is validated using a finite-element model of an induction motor and also by laboratory tests using commercial cage motors.

Good agreement was obtained between theoretical analysis, simulation and experimental results, proving that the proposed approach constitutes a useful tool for the study and diagnostics of double and multiple bar breakages.

\section{REFERENCES}

[1] W. Deleroi "Broken bar in a squirrel-cage rotor of an induction motor Description by superimposed fault-currents", Archiv für elektrotechnik 67 (1984) pp.91-99

[2] I. Kerzenbaum and C.F. Landy, "The Existence of Large Inter-Bar Currents in Three Phase Squirrel Cage Motors with Rotor-Bar and/or Endring Faults", IEEE Transactions on Power Apparatus and Systems, Volume PAS-103, Issue 7, July 1984, pp 1854-1862.
[3] J. Pennman and A. Stavrou, "Broken rotor bars: their effect on the transient performance of induction machines", IEE Proc-Electr. Power Appl., Vol. 143, No 6, November 1996 pp 449-457

[4] F. Filippetti, G. Franceschini, C. Tassoni, P. Vas, "AI Techniques in induction machines diagnosis including the speed ripple effect", IEEE Transactions on Industry Applications, Vol. 34, No 1, January/February 1998, pp 98-108.

[5] C. H. Rojas, M. G. Melero, M. F. Cabanas, G. A. Orcajo, M. P. Donsión, J. Solares, "Analysis by the finite element method of the influence caused in the current distribution in rotor squirrel-cage induction motor and current spectrum by the number and distribution of faulty bars during a rotor failure", Proceedings of the SDEMPED'01 - The IEEE International Symposium on Diagnostics of Electrical Machine, Power Electronics and Drives, Grado - Italy, 2001, pp 479 - 486, Vol 1.

[6] C. Hargis, B.G. Gaydon, K. Kamash., "The detection of rotor defects in induction motors," Proceedings of the 1982 IEE International Conference Electrical Machines. Design and Application, London (UK), 1982, pp. 216-220.

[7] M. H. Benbouzid, "A review of induction motors signature analysis as a medium for fault detection" IEEE Transactions on Industrial Electronics, Vol. 47, No. 5, October 2000. pp. 984-993

[8] A. Patyck "Application of forward/backward components to the analysis of failure states of the squirrel cage motor. Part 2: calculation of the current flow distribution on the squirrel cage and the stator and of the components of the electromagnetic moment". Archiv für elektrotechnik 69 (1986) pp.439-443

[9] H.A. Toliyat, T.A. Lipo, "Transient Analysis of Cage induction motors under stator Rotor Bar and end-ring Faults", IEEE Trans. on Energy Conversion, Volume:10, Issue:2,June1995. pp. 241-247

[10] T.J. Sobczyk, W. Maciolek "Does the component (1-2s)f0 in stator current is sufficient for detection of rotor cage faults?" Proc. International Symposium on diagnostic for electrical machines, power electronics and Drives, SDEMPED 2005, Vienna, September 2005. CDROM

[11]Menacer，A.Moreau，S. Champenois，G. Said，M.S.N. Benakcha，A. 'Experimental Detection of Rotor Failures of Induction Machines by Stator Current Spectrum Analysis in Function of the Broken Rotor Bars Position and the Load' EUROCON, 2007. The International Conference on 'Computer as a Tool' Sept. 2007,pp. 1752-1758

[12] Jawad Faiz *, B.M. Ebrahimi, 'Locating rotor broken bars in induction motors using finite element method' Energy Conversion and Management Vol.50, Issue 1, January 2009, pp. 125-131.

[13] Demerdash, N.A. O.; Sayed-Ahmed, A.; Sizov, G.Y.; Yeh, C.; 'Analysis and Diagnostics of Adjacent and Nonadjacent Broken Rotor Bar Faults in Squirrel-Cage Induction Machines' IEEE Transactions on Industrial Electronics, Accepted for future publication.Digital Object Identifier 10.1109/TIE.2008.2011341

[14] Henao, H.; Razik, H.; Capolino, G.-A, "Analytical approach of the stator current frequency harmonics computation for detection of induction machine rotor faults" IEEE Transactions on Industry applications Volume 41, Issue 3, May-June 2005 Page(s):801 - 807

[15] A. Bellini, F. Filippetti, G.Franceschini, G.B.Kliman, "Quantitative Evaluation of Induction Motor Broken Bars by Means of Electrical Signature Analysis", IEEE Transactions On Industry Applications, Vol. 37, No. 5, September/October 2000, pp.1248-1255

[16]Concari,C. Franceschini,G.Lorenzani, E.Toscani,A. "Severity assessment of rotor faults in closed Loop induction drives by different approaches" IEEE International Symposium on Diagnostics for Electric Machines, Power Electronics and Drives, SDEMPED Sept. 2007 pp 309-315.

[17] J.Stepina "Fundamental equations of the space vector analysis of electrical machines". Acta technica ČSAV, №2, 1968, pp.184-198

[18] Vas,Peter,"Parameter estimation, condition monitoring, and diagnosis of electrical machines",Oxford : Clarendon Press , 1993

[19] G.B Kliman, J Stein and R.D. Endicott, "Noninvasive Detection of Broken Rotor Bars in Operating Induction Motors", IEEE Transactions on Energy Conversion, Vol. 3, No. 4, December 1988

[20] M. Riera, J. Antonino-Daviu, J. Roger-Folch and M.P. Molina, “The Use of the Wavelet Approximation Signal as a Tool for the Diagnosis of Rotor Bar Failures," IEEE Transactions on Industry Applications , Vol. 44, No. 3, May-June 2008, pp. 716-726.

[21] C. Rojas, M. G. Melero, M. F. Cabanas, J. M. Cano, G. A. Orcajo, F. Pedrayes, "Finite Element model for the study of inter-turn short-circuits in induction motors", Proceedings of the SDEMPED'07 - The IEEE International Symposium on Diagnostics of Electrical Machine, Power Electronics and Drives, Cracow - Poland, 2007, pp $415-419$. 